\title{
Características del consumo de filgrastim en el servicio de oncología del Hospital Ramiro Priale Priale de EsSalud.
}

\author{
Characteristics of filgrastim consumption in the oncology service of the Ramiro Priale Priale Hospital \\ EsSalud.
}

\begin{abstract}
Maribid Kely Nuñez Julcarima ${ }^{1, a}$, Araceli Mirelly Julcarima Naupari 1,a, Vilma Amparo Junchaya Yllescas ${ }^{1, b}$
\end{abstract}

\section{RESUMEN}

Objetivo: Determinar las características del consumo de Filgrastim en el Servicio De Oncología del Hospital Ramiro Priale Priale de EsSalud. Material y métodos: Corresponde a un estudio descriptivo, retrospectivo y de corte transversal, empleándose una muestra de 144 casos clínicos del consumo de Filgrastim; empleándose como técnica de análisis documental y el instrumento la ficha de recolección de datos. Resultados: La prevalencia de utilización del filgrastim en la práctica clínica en el Servicio de Oncología del Hospital Ramiro Priale Priale de EsSalud, fue del $100 \%$, con un consumo según edad, entre los rangos de 68 a 77 años, mayormente en el sexo femenino, con promedio mensual de consumo de 52.31 unidades y costo promedio anual de 94,221.6 y mensual 7851.80 nuevos soles.

Conclusiones: El consumo de Filgrastim 300mg, fue mayor en mujeres y pacientes de la tercera edad, resaltando el diagnóstico Linfoma No Hodgkin difuso para su consumo y se requiere se implemente guías de manejo para su prescripción.

PALABRAS CLAVE: Consumo, filgrastim, oncología, hospital.

\section{SUMMARY}

Objective: Yo determine the characteristics of service consumption of Filgrastim Oncology Hospital Ramiro Priale Prialé of EsSalud. Material and Methods:It is a descriptive, retrospective study and cross-section, using a sample of 144 cases of consumption of Filgrastim; using as documentary analysis technique and instrument data collection sheet. Results: The prevalence of use of filgrastim in clinical practice in the service of Oncology the Hospital Ramiro Priale Prialé of EsSalud, was $100 \%$, with a consumption according to age, between ranges from 68 to 77 years, mostly in the female sex, with monthly average of consumption of 52.31 units and annual average cost of 94,221.6 and monthly 7851.80 new soles. Conclusions: Consumption of Filgrastim 300mg, was higher in women and older patients, highlighting the lymphoma non-Hodgkin's diffuse diagnosis for consumption and is required for your prescription management guidelines to be implemented.

KEYWORDS: Consumption, filgrastim, oncology, hospital. 


\section{INTRODUCCIÓN}

En el contexto global de la medicina, los medicamentos constituyen un elemento con características especiales; en primer lugar por su papel como parte de la asistencia médica y en segundo lugar, por el valor que tiene conocer el modo como son utilizados en la práctica médica, tanto es así que la base para conseguir el uso racional de medicamentos está constituida por el conocimiento de la utilización de los mismos y el análisis de sus variaciones de prescripción a lo largo del tiempo, particularmente de aquellos medicamentos de grupos farmacológicos de gran consumo y de aquellos en los que aparecen innovaciones terapéuticas (1).

La Organización Mundial de la Salud (OMS) ha reconocido la necesidad de establecer una política nacional de medicamentos y la importancia de una estrategiaasociadadeinvestigaciónqueincluyaestudios de utilización de medicamentos (EUM); considerado como un tema prioritario que tiene por objeto de análisis: estudiar la comercialización, distribución, prescripción y uso de los medicamentos en una sociedad, con acento especial sobre las consecuencias médicas, sociales y económicas resultantes; los cuales abarcan: la prescripción, dispensación e ingesta de medicamentos, y son la principal herramienta para detectar la mala utilización, identificar los factores responsables, diseñar intervenciones efectivas de mejora, y evaluar los logros de esas intervenciones. Representan un avance en el conocimiento de la calidad y eficiencia de la atención sanitaria. Además de tener un carácter interdisciplinario, con tareas específicas para médicos, farmacéuticos, salubristas, economistas, informáticos y otros profesionales. Tales estudios implican la recolección de datos relevantes sobre el uso de los medicamentos, su organización y análisis y finalmente la toma de decisiones adecuadas destinadas a un uso racional de los mismos $(2,3)$.

Por otro lado, el cáncer es un problema de salud pública a escala mundial, pues así lo demuestran sus altas tasas de incidencia y mortalidad. Un informe publicado en 2007 por la American Cáncer Society, indica que aproximadamente $15 \%$ de todos los casos están vinculados a infecciones de estómago, hígado y cáncer cervico uterino.

Se relaciona directamente a fumar cigarrillos, mayor consumo de grasas saturadas y menor actividad física. Asimismo, se estima que cerca de veinte millones de personas presentarán algún tipo de cáncer cada año, de las cuales cerca de nueve millones corresponderán a casos incidentes. E n Latinoamérica el cáncer ocupa el tercer lugar de las causas de muerte y en el Perú, el Registro de Cáncer de Lima Metropolitana, ha publicado tasas de incidencia y mortalidad por cáncer globales de 150.7 y 78.3 por cien mil habitantes de nuestra ciudad capital $(3,4)$.

El Filgrastim es el factor estimulante de colonias granulocíticas humano (GCSF), producido por tecnología de ADN recombinante. Es una proteína de 175 aminoácidos con un peso molecular de 18.800 daltons, se produce a través de ingeniería genética, por la inserción del gen humano del G-CSF a una bacteria, la escherichia-coli. La secuencia de aminoácidos es idéntica a la humana, salvo por la adición de metionina en extremo aminoterminal y por la falta de glicosilación. Éste, cuando es aplicado a enfermos sometidos a quimioterapia, reduce significativamente la incidencia, gravedad y duración de la neutropenia. También, reduce el número de ingresos hospitalarios, disminuyendo además el período de hospitalización $(5,6)$.

El uso de factores estimulantes de colonias granulocíticas (G-CSF) en combinación con pautas de quimioterapia intensiva es una indicación aceptada para el tratamiento coadyuvante para diversos tipos de cáncer. Recientemente, la Food and Drug Administration (FDA) americana los ha aprobado para su uso clínico, con los nombres genéricos de Filgrastim (7).

El objetivo del presente trabajo fue determinar las características del consumo de Filgrastim en el Servicio De Oncología del Hospital Ramiro Priale Priale de EsSalud.

\section{MATERIAL Y MÉTODOS}

El tipo de investigación fue básica y de nivel descriptivo, retrospectivo y transversal.

La población de estudio corresponde a los casos clínicos del servicio de oncología del Hospital de EsSalud Ramiro Priale Priale que consumieron Filgrastim y que corresponde en aproximadamente 225 pacientes.

\section{Criterios de inclusión}

Casos clínicos de pacientes que corresponda al servicio de Oncología. 
Casos clínicos de pacientes que sean de los meses de enero a diciembre del 2018.

Casos clínicos que sean de pacientes adultos mayores a 18 años.

\section{Criterios de exclusión}

Casos Clínicos de pacientes que no corresponda al servicio de oncología.

Casos clínicos que sean del presente año.

Casos clínicos que sean de pacientes menores de edad.

El instrumento utilizado en esta investigación fue la Ficha de recolección de datos.

En este instrumento, fue plasmado por escrito información importante sobre el consumo del filgrastim, tomándose en consideración las dimensiones de la investigación como:

- Prevalencia

- Consumo

- Efectividad del consumo

- Consumo anual x 100 camas

- Costo consumo anual

\section{RESULTADOS}

De la práctica clínica en el Servicio de Oncología del Hospital Ramiro Priale Priale de EsSalud, se puede apreciar en la tabla 1 una prevalencia del 100 $\%(n=144)$ de los pacientes que recibieron tratamiento con filgrastim.

Figura 1. Prevalencia del consumo de filgrastim en la práctica clínica en el Servicio de Oncologia del Hospital Ramiro Priale Priale de EsSalud.

\begin{tabular}{lcc}
\hline Prevalencia & $\mathbf{n}$ & $\mathbf{\%}$ \\
\hline SI & 144 & 100 \\
NO & 0 & 0 \\
TOTAL & 144 & 100 \\
\hline
\end{tabular}

Tabla 2. Distribución del consumo de filgrastim según sexo en el Servicio de Oncologia del Hospital Ramiro Priale Priale de EsSalud

\begin{tabular}{lcc}
\hline Sexo & $\mathbf{n}$ & $\mathbf{\%}$ \\
\hline Femenino & 92 & 64 \\
Masculino & 52 & 36 \\
TOTAL & 144 & 100 \\
\hline
\end{tabular}

De la práctica clínica en el servicio de Oncología del Hospital Ramiro Priale Priale de EsSalud, se puede apreciar en la tabla 2 que un $64 \%(n=92)$, corresponden al sexo femenino, mientras que un $36 \%$ $(\mathrm{n}=52)$, pertenecen al sexo masculino de los pacientes que consumen el tratamiento con filgrastim.

De la práctica clínica en el servicio de Oncología del Hospital Ramiro Priale Priale de EsSalud, se puede apreciar que en la tabla 3 que un $38 \%(n=54)$ son pacientes mayores a 77 años. Además, se aprecia que un $27 \%(n=39)$, sus edades se encuentran entre 68 a 77 años de edad. También se observa que un $18 \%(\mathrm{n}=26)$ sus edades se encuentran entre 48 a 57 años. Otro 12 $\%(\mathrm{n}=17)$ de la muestra de estudio, se encuentran entre las edades de 58 a 67 años. Se aprecia que un 5\% $(\mathrm{n}=7)$, sus edades de los pacientes se encuentran entre 38 a 47 años. Finalmente, un $1 \%(n=1)$ sus edades de los pacientes se encuentran entre 28 a 37 años.

Tabla 3. Distribución del consumo de filgrastim según edad en el Servicio de Oncologia del Hospital Ramiro Priale Priale de EsSalud

\begin{tabular}{lcc}
\hline Edad & n & \% \\
\hline De 18 a 27 años & 0 & 0 \\
De 28 a 37 años & 1 & 1 \\
De 38 a 47 años & 7 & 5 \\
De 48 a 57 años & 26 & 18 \\
De 58 a 67 años & 17 & 12 \\
De 68 a 77 años & 39 & 27 \\
Mayores de 77 años & 54 & 38 \\
\hline TOTAL & 144 & 100
\end{tabular}

En la tabla 4, se puede apreciar que un $27,08 \%$ $(n=39)$, padecen del diagnóstico Linfoma no hodgkin difuso.También se encuentra que un $21,53 \%(n=31)$ padecen la muestra en estudio de otros diagnósticos. Así también un $13,89 \% \quad(\mathrm{n}=20) \quad$ corresponde Tumor maligno de la mama/parte no especificada. Igualmente, otro $12,50 \%,(\mathrm{n}=18)$ Tumor maligno del estómago /parte no especificada. Otro valor relevante $9,72 \%(\mathrm{n}=14)$, el diagnóstico encontrado fue cáncer de páncreas. También un 4,86\% $(\mathrm{n}=7)$, fue encontrado en la muestra de estudio,el diagnóstico tumor maligno del ano.

En la tabla 5, se puede observar que el consumo promedio de tendencia anual fue de 629 unidades y siendo el promedio anual de 52,41 unidades del filgrastim. 
Tabla 4. Distribución del consumo de filgrastim según Diagnóstico en el Servicio de Oncologia del Hospital Ramiro Priale Priale de EsSalud.

\begin{tabular}{lcc}
\hline Diagnóstico & $\mathbf{n}$ & $\mathbf{\%}$ \\
\hline Linfoma no hodgkin difuso & 39 & 27.8 \\
Leucemia linfoblástica & 3 & 2.08 \\
Tumor maligno de la mama / & 20 & 13.89 \\
$\begin{array}{l}\text { Parte no especificada } \\
\text { Tumor maligno del estómago/ }\end{array}$ & 18 & \\
Parte no especificada & & \\
Mieloma múltiple & 4 & \\
Tumor maligno del tejido & 4 & 2.78 \\
conjuntivo y tejido blando & & \\
Anemia aplásica & 4 & 2.78 \\
Tumor malgno del ano & 7 & 4.86 \\
Cáncer del páncreas & 14 & 9.72 \\
Otros diagnóstico & 31 & 21.53 \\
TOTAL & 144 & 100 \\
\hline
\end{tabular}

Tabla 5. Distribución del consumo promedio del filgrastim según tendencia anual en el Servicio de Oncologia del Hospital Ramiro Priale Priale de EsSalud.

\begin{tabular}{lcc}
\hline Tendencia anual & $\begin{array}{c}\text { Consumo mensual } \\
\text { DDD/28 camas }\end{array}$ & Promedio \\
\hline Enero & 53.91 & \\
Febrero & 54.16 & \\
Marzo & 54.83 & \\
Abril & 56.58 & \\
Mayo & 57.91 & 52.31 \\
Junio & 57.08 & \\
Julio & 57.25 & \\
Agosto & 55.75 & \\
Setiembre & 53.25 & \\
Octubre & 44.75 & \\
Noviembre & 42.91 & \\
Diciembre & 39.83 & \\
TOTAL & 628.21 & \\
\hline
\end{tabular}

Se encontró que el consumo promedio del mes de enero y febrero fue de 54 unidades para cada mes. También se encontró que el consumo promedio del mes de Marzo fue de 55 unidades. De igual forma se encontró que en los meses de abril, junio y Julio el consumo promedio del mes para cada caso fue de 57 unidades.Además, se encontró que en el mes de agosto 
Tabla 6. Distribución del costo promedio del filgrastim según tendencia anual en el Servicio de Oncologia del Hospital Ramiro Priale Priale de EsSalud.

\begin{tabular}{lcc}
\hline $\begin{array}{l}\text { Tendencia } \\
\text { costo anual }\end{array}$ & $\begin{array}{c}\text { Costo } \\
\text { Consumo mensual } \\
\text { Soles }\end{array}$ & $\begin{array}{c}\text { Promedio } \\
\text { Costo } \\
\text { Consumo anual }\end{array}$ \\
\hline Enero & 8042.8 & \\
Febrero & 8080.15 & \\
Marzo & 8192.2 & \\
Abril & 8441.2 & \\
Mayo & 8652.85 & \\
Junio & 8515.9 & \\
Julio & 8540.6 & \\
Agosto & 8304.05 & \\
Setiembre & 7955.45 & \\
Octubre & 7133.75 & \\
Noviembre & 6411.65 & \\
Diciembre & 5951 & \\
TOTAL & 94221.6 & \\
\hline
\end{tabular}

En la tabla 6, se puede apreciar que el costo promedio del consumo anual fue de 94,221.6 nuevos de enero a diciembre del 2018 y siendo el promedio mensual 7851.80. En el mes de enero, el gasto de consumo promedio del filgrastim fue de 8042.8, nuevos soles. En el mes de febrero, el gasto de consumo promedio del filgrastim fue de 8080.15 , nuevos soles. En el mes de marzo, el gasto de consumo promedio del filgrastim fue de 8192.2, nuevos soles. En el mes de abril, el gasto de consumo promedio del filgrastim fue de 8441.2, nuevos soles. En el caso del mes de mayo el gasto promedio fue de 8652.85 , nuevos soles. También se tuvo un gasto en el mes de junio de 8515.9, nuevos soles. De tratarse del mes de Julio el gasto ocasionado fue de 8540.6 nuevos soles. En el mes de agosto el gasto fue de 8304.05 nuevos soles. En el caso del mes de septiembre se gastó 7955.45 nuevos soles. En octubre el gasto promedio del mes de octubre fue de 7133.75 nuevos soles. En noviembre el gasto del consumo del mes fue de 6411.65, nuevos soles. En diciembre el gasto del consumo del filgrastim fue de 5951 nuevos soles.

\section{DISCUSIÓN}

En el estudio realizado, después de la revisión de 144 casos clínico de enero a diciembre del año 2018 y correspondiente a la práctica clínica en el servicio de Oncología del Hospital Ramiro Priale Priale de EsSalud, se puede apreciar en la tabla 1 una prevalencia del $100 \%(\mathrm{n}=144)$ de los pacientes que recibieron tratamiento con Filgrastim, lo que se corrobora con el estudio de Cenizario, quien encuentra que, la prevalencia del consumo de Filgrastim fue de $88.31 \%(15)$.

Otro de los resultados encontrados en este estudio es que un $64 \%(\mathrm{n}=92)$, corresponden al sexo femenino, mientras que un $36 \%(n=52)$, pertenecen al sexo masculino de los pacientes que consumen el tratamiento con Filgrastim, lo que se corrobora con el trabajo de Miranda quien concluye que, de 635 pacientes del Servicio de OncoHematología un $67.36 \%$ de pacientes que corresponden al sexo femenino y $32.64 \%$ al sexo masculino (9), también se asemeja a la investigación de Zapata, quien en su investigación encontró que el $54 \%$ pertenece al sexo femenino y el $46 \%$ al sexo masculino (10).

Así también, en este trabajo fue importante demostrar que de acuerdo a la práctica clínica en el servicio de Oncología del Hospital Ramiro Priale Priale de EsSalud, la edad de los pacientes de los casos clínicos, se encuentra un $38 \%(\mathrm{n}=54)$ son pacientes mayores a 77 años, seguido de un $27 \%(n=39)$, sus edades se encuentran entre 68 a 77 años de edad, lo 
que difiere con el estudio de Tchekmeyán, quien halló que de 243, la edad promedio fue de 55 años (11).

Además, en este trabajo fue encontrado que el diagnóstico Linfoma No Hodgkin difuso, represento un $27,08 \%(n=34)$, para el mayor consumo del tratamiento con Filgrastim, lo que se contrapone con el trabajo de Cenizario, quien concluye que el consumo fue mayor en la leucemia linfocitica aguda, que representó el diagnóstico de mayor consumo (8), también difiere con el trabajo Miranda, quien demostró que la enfermedad Tumor Maligno de la Mama, Parte no Especificada con 32.41\% representó el diagnóstico de mayor consumo del Filgrastim (9), de igual forma difiere con el trabajo de Zapata, quien encontró que el diagnóstico de mayor consumo de este medicamento pertenece a Leucemia Linfoblástica con 32.14\% (10). Otro de los resultados hallados en esta investigación fue que el consumo promedio de tendencia anual del Filgrastim en 144 casos clínicos fue de 629 unidades y siendo el promedio anual de 52,41 por 28 camas, lo que se corrobora de cierta manera con el trabajo de Cenizario, quien concluye que el promedio anual del consumo expresado en DDP/100camas-día fue de $43.83(8)$.

Además, se hallaron como resultado en este estudio que el costo promedio del consumo anual fue de 94,221.6 nuevos de enero a diciembre del 2018, como inversión en la prescripción del Filgrastim de $300 \mathrm{mg}$, lo que se asemeja al trabajo de Cenizario, quien demostró que el costo anual de Filgrastim fue de S/.98 598.03 nuevos soles(8); sin embargo se contrapone al estudio de Miranda, quien demuestra que el costo del consumo anual del Filgrastim de300mcg/dl fue: S/.60298, 81 nuevos soles (9) ; de igual forma con el trabajo de Zapata), que el año 2013, encontró que el costo del consumo anual de Filgrastim $300 \mathrm{mcg} / \mathrm{ml}$ fue de $\mathrm{S} / .12688 .13$ nuevos soles (10).

Por esta razón el Químico Farmacéutico, como miembro integrante del equipo de salud, está capacitado para conservar la salud y prevenir las enfermedades, desempeñándose en diferentes campos en los que está incluido el uso adecuado de medicamentos y establecer guías de manejo del Filgrastim en sus protocolos de quimioterapia en el área de oncología y hematología clínica.

\section{CONCLUSIONES}

El consumo de Filgrastim 300mg, fue mayor en mujeres y pacientes de la tercera edad, resaltando el diagnóstico Linfoma No Hodgkin difuso para su consumo y que requiere que se implemente guías de manejo para su prescripción.

La prevalencia de utilización del filgrastim en la práctica clínica en el servicio de Oncología del Hospital Ramiro Priale Priale de EsSalud fue del 100\%.

El consumo de filgrastim según edad, fue entre los rangos de 68 a 77 años y en más de la mitad de sexo femenino.

El consumo de Filgrastim fue mayor en el caso clínico de diagnóstico Linfoma no hodgkin difuso en el 27,08 $\%(n=39)$.

El consumo promedio anual de Filgrastim en el servicio de oncología del Hospital Ramiro Priale Priale de EsSalud, no mostró una tendencia creciente ni decreciente significativa durante el periodo de estudio y fue de 52.31 unidades.

El costo promedio del consumo anual de Filgrastim en el servicio de Oncología del Hospital Ramiro Priale Priale de EsSalud, fue de94,221.6 y siendo el promedio mensual 7851.80 .

\section{Correspondencia}

Vilma Amparo Junchaya Yllescas Av. Giráldez 542, Huancayo 12001. Junin, Perú

\section{REFERENCIAS BIBLIOGRÁFICAS}

1. Furones J, Mederos A, Cordero A, Cruz M, López A. Caracterización de los Estudios de Utilización de Medicamentos publicados en revistas cubanas, 19902003. Rev Cubana Farm. 2006;40(1):1-8.

2. Arnello M, Quintana J, Barraza P. Neutropenia Febril en Niños con Cáncer en un Centro Asistencial en Santiago, Chile. Rev Chil Infect.2007; 24(1): 2732.

3. Ministerio De Salud. Informe Técnico $\mathrm{N}^{0} 11$ $-2008 \quad$ Filgrastim $300 \mu \mathrm{cg} / \mathrm{Ml}, \quad$ Filgrastim $480 \mu \mathrm{cg} / \mathrm{Ml}$ (Inyectable).Perú: Dirección General De Medicamentos Insumos Y Drogas; 2008.

4. Álvarez F. Farmacoepidemiología. Estudios de Utilización de Medicamentos. Parte I: Concepto y Metodología. Farmacoter. 2004;2(3) 129-36.

5. López B, López A, Cabeza A, et al. Evolución de los Estudios de Utilización de Medicamentos: del Consumo a la Calidad de la Prescripción. Cuadernos económicos de ICE. 2004; 67:161-90.

6. López M, Jiménez R, Borbolla J, et al .Factor estimulante de colonias granulocitos en el tratamiento de neutropenia febril. Gac Méd Méx.2000; 136 (2): 99-105.

7. Escobar I, Sevilla E, Rodriguez M, et al. 
Estudio multicéntrico de utilización de factores estimulantes de crecimiento hematopoyético. Farm Hosp.1998;22(3):109-25.

8. Garjon F. Influencia del horario de administración en la efectividad del Filgrastim en pacientes oncológicos. Rev Farm Hosp. 1997; 21 (3): 133-6.

9. Bello A. Estudio de evaluación de la Calidad de la prescripción y costo del factor estimulante de colonias de granulocitos, Filgrastim; en el paciente Onco-Hematológico, Hospital base de Valdivia; periodo 2003 a 2008.Tesis. Chile: Universidad Austral de Chile; 2010.
10. Homero C, Garza F, Cantú O, et al. Comparación de la efectividad de la movilización de células hematopoyéticas con quimioterapia y filgrastim, o con filgrastim solo, para autotrasplante en pacientes con linfoma. Rev Gac Med Mex. 2016;152: 57-65.

11. La Fuente M, Velaz M, Mejías A, Saavedra M, Diez A. Uso de filgrastim en pacientes con cáncer de mama y esquema de quimioterapia FEC600. Atención farmaceútica. 2004;6(5):319-24.

Recibido: $14 / 02 / 2020$

Aceptado: 15/06/2020 